\title{
Dietary Intakes in Obese Pregnancy
}

\author{
K. Lindsay ${ }^{1}$, B. McNulty ${ }^{2}$, L. Brennan ${ }^{2}$ and F. McAuliffe ${ }^{1}$ \\ ${ }^{1}$ UCD Department of Obstetrics and Gynaecology, National Maternity Hospital, Holles Street, Dublin 2 and ${ }^{2}$ UCD School \\ of Agriculture and Food Science, University College Dublin, Ireland
}

Currently, in Ireland, it is estimated that $15 \%$ of our obstetric population has a body mass index (BMI) in the obese category ${ }^{(1)}$. Maternal obesity significantly increases the risk of various complications such as preeclampsia, gestational diabetes, emergency caesarean section, stillbirth and macrosomia ${ }^{(2)}$. Pregnancy is often considered an opportune time to encourage healthy lifestyle behaviours among expectant mothers who may be more motivated than at other stages in the lifecycle. Although intervention studies have demonstrated effectiveness of dietary and lifestyle interventions to improve obstetric outcomes ${ }^{(3)}$, there is a paucity of literature examining the habitual dietary intakes of obese pregnant women. Such data is important in order to develop tailored dietary advice for these women who are at high risk of adverse outcomes. The aim of the current study is to assess the dietary intakes of obese pregnant women and compare them to dietary recommendations.

One hundred women with an early pregnancy BMI of $30.0-39.9 \mathrm{~kg} / \mathrm{m}^{2}$ were recruited at their first antenatal visit in the National Maternity Hospital, Dublin. Dietary intakes were assessed in the second trimester using a 3-day food diary and data was entered into WISP for nutrient intake analysis. Nutritional supplement usage was recorded and underreporting of energy intake was assessed using a Goldberg ratio of $0.9^{(4)}$. Nutrient intakes were compared to Irish recommended dietary allowances ${ }^{(5)}$ and compliance was assessed by calculating the percentage of the population whose mean intake achieved the recommendation.

Twenty-seven per cent of women were identified as under-reporters of energy intake and were excluded from further analyses. The mean age of the remaining 73 women was 31 years, $81 \%$ were of Irish ethnicity and $47 \%$ were primiparous. Antenatal use of supplements containing folic acid, iron and vitamin D were reported as 71,75 and $68 \%$ respectively.

\begin{tabular}{|c|c|c|c|c|}
\hline Nutrient & Mean & SD & Recommended Intake & $\%$ Compliance \\
\hline Energy KJ & 8140 & 1459 & & \\
\hline Protein g/day ( $\%$ energy) & $86.5(18.1)$ & $15.8(3.2)$ & $>10 \%$ energy & 100 \\
\hline Carbohydrate g/day (\% energy) & $220.2(45.7)$ & $42.4(6.1)$ & $>55 \%$ energy & 19.2 \\
\hline Total fat g/day ( $\%$ energy) & $80.4(37.0)$ & $22.2(5.7)$ & $<35 \%$ energy & 79.5 \\
\hline Saturated fat g/day ( $\%$ energy) & $32.9(15.1)$ & $10.5(3.3)$ & $<10 \%$ energy & 12.3 \\
\hline Calcium $\mathrm{mg} /$ day & 969.6 & 316.8 & $1200 \mathrm{mg} /$ day & 49.3 \\
\hline Iron mg/day & 11.1 & 3.1 & $15 \mathrm{mg} /$ day & 28.8 \\
\hline Vitamin $D \mu \mathrm{g} /$ day & 3.2 & 2.5 & $10 \mu \mathrm{g} / \mathrm{day}$ & 6.8 \\
\hline Folate $\mu \mathrm{g} /$ day & 299.9 & 109.4 & $500 \mu \mathrm{g} /$ day & 12.3 \\
\hline Vitamin $B_{12} \mu \mathrm{g} /$ day & 4.8 & 2.0 & $1.6 \mu \mathrm{g} / \mathrm{day}$ & 100 \\
\hline
\end{tabular}

Obese pregnant women consume high amounts of saturated fat and inadequate carbohydrate. Their overall compliance to recommended intakes of the key pregnancy micronutrients is poor, although similar inadequate intakes have been reported among the general Irish obstetric population $^{(6)}$. Supplement usage may compensate for inadequate micronutrient intakes but further research into the actual foods consumed by obese pregnant women would aid the development of tailored and effective dietary interventions that could improve their overall nutrient intake profile.

1. McGowan CA \& McAuliffe FM (2012) Eur J Clin Nutr 66, 906-913.

2. Ovesen P, Rasmussen S \& Kesmodel U (2011) Obstet Gynecol 11, 305-312.

3. Thangaratinam S, Rogozińska E, Jolly K et al. (2012) BMJ 16, 2088-2103.

4. Goldberg GR, Black AE, Jebb SA et al. (1991) Eur J Clin Nutr. 45, 469-581.

5. Food Safety Authority of Ireland (1999) Recommended Dietary Allowances for Ireland. FSAI: Dublin.

6. McGowan CA \& McAuliffe FM (2013) Pub Health Nutr 16, 97-107. 